\section{Aspectos epidemiológicos da coinfecção Tuberculose/HIV no Brasil: revisão integrativa}

\author{
Epidemiological aspects of TB/HIV coinfection in Brazil: integrative review \\ Aspectos epidemiológicos de la coinfección Tuberculosis/VIH (TB/VIH) en Brasil: revisión integrativa
}

Lihuan Oliveira de Miranda ${ }^{1}$, Gustavo Bacelar Fontenele Araújo ${ }^{1}$, Daniela Furtado Rodrigues de Andrade $^{2}$, Mariana Lustosa Carvalho ${ }^{3}$, Sávio Miranda Fontineles da Silva ${ }^{2}$, Anderson de Melo Moreira², Daniela Reis Joaquim de Freitas ${ }^{2}$

1. Universidade Federal do Piauí. Departamento de Medicina. Teresina, Piauí, Brasil.

2. Universidade Federal do Piauí. Programa de Pós-graduação em Ciências da Saúde. Teresina, Piauí, Brasil.

3. Universidade Federal do Piauí. Programa de Pós-graduação em Enfermagem. Teresina, Piauí, Brasil.

\begin{abstract}
Objective: To analyze in literature the epidemiological aspects of tuberculosis/HIV coinfection in Brazil. Method: Descriptive research of the type integrative review of the literature with analysis of data processed by iramuteq software. The search was performed in the databases BVS, LILACS, MEDLINE and PUBMED with articles available in full published eletronically between 2013-2017. Results: 23 articles were founded. The coinfection is more prevalent among males, black color/race, low income, urban residents aged 20-50 years. Morbidity and mortality in coinfection is high and it is more prevalent in the southern and southeastern regions of Brazil. Conclusion: It can be concluded that a large number of HIV-positive individuals develops HIV/TB coinfection and one of the major problems in the treatment of both is non-adherence and/or abandonment, which increase therapeutic failures and drug resistance.
\end{abstract}

Descriptors: Tuberculosis; HIV; Coinfection; Brazil

\section{RESUMO}

Objetivo: Analisar, a partir da literatura, os aspectos epidemiológicos da coinfecção Tuberculose/HIV (TB/HIV) no Brasil. Método: Trata-se de um revisão integrativa. A busca foi realizada nas bases de dados BVS, LILACS, MEDLINE e PubMed com artigos disponíveis na integra de forma eletrônica publicados entre os anos de 2013 e 2017. Resultados: Foram selecionados 23 estudos originais. Segundo os achados, a coinfecção é mais prevalente entre indivíduos do sexo masculino, negros, de baixa renda, residentes urbanos com idade entre 20 e 50 anos. A morbidade e mortalidade na coinfecção é alta e possui maior prevalência na região sul e sudeste do Brasil. Conclusão: Grande número de HIV positivos desenvolve a coinfecção HIV/TB, e um dos maiores problemas no tratamento de ambas é a não adesão e/ou o abandono do mesmo, que incrementam as falhas terapêuticas e a resistência às drogas utilizadas.

Descritores: Tuberculose; HIV; Coinfecção; Brasil.

\section{RESUMÉN}

Objetivo: Analizar en la literatura los aspectos epidemiológicos de la coinfección Tuberculosis/VIH (TB/VIH) en Brasil. Método: Investigación descriptiva del tipo revisión integrativa de la literatura con análisis de datos procesados por el software IRAMUTEC. La búsqueda fue realizada en las bases de datos BVS, LILACS, MEDLINE y PUBMED con artículos disponibles en la integración de forma electrónica publicados entre 2013-2017. Resultados: Se han encontrado 23 artículos. La coinfección es más prevalente entre individuos del sexo masculino, color/raza negra, de baja renta, residentes urbanos con edad entre 20-50 años. La morbilidad y mortalidad en la coinfección es alta y tiene mayor prevalencia en la región sur y sudeste de Brasil. Conclusión: Se puede concluir que gran número de VIH positivos desarrolla la coinfección VIH/TB, y uno de los mayores problemas en el tratamiento de ambas es la no adhesión y/o el abandono del mismo, que incrementan las fallas terapéuticas y la resistencia a las drogas utilizadas.

Descriptores: Tuberculosis; VIH; Coinfección; Brasil.

\section{Como citar este artigo:}

Miranda LO, Araujo GBF, Carvalho ML, Silva SMF, Andrade DFR, Moreira AM, et al. Aspectos epidemiológicos da coinfecção Tuberculose/HIV no Brasil: revisão integrativa. Rev Pre Infec e Saúde.2017;3(3):59-70. Available from: http://www.ojs.ufpi.br/index.php/nupcis/article/view/5848 


\section{INTRODUÇÃO}

A introdução de terapia anti-retroviral altamente ativa (HAART) levou a um aumento da sobrevivência entre os pacientes infectados pelo HIV e melhorou a qualidade de vida das pessoas vivendo com HIV/AIDS ${ }^{1}$. No entanto, a tuberculose (TB) continua a ser a infecção oportunista mais comum e uma das principais causas de morte entre os pacientes com HIV. Em todo o mundo, estima-se que haja 10,4 milhões casos de TB, $13 \%$ dos quais em coinfecção com HIV2. No Brasil, foram detectados 66.796 novos casos de tuberculose e 4.543 mortes ocorreram em 2016 e ocupa a $1^{\text {a }}$ causa de mortes dentre as doenças infecciosas definidas dos pacientes com HIV/AIDS ${ }^{3}$.

A tuberculose associada ao HIV alterou as perspectivas de controle da tuberculose no mundo, levando a um aumento na incidência da tuberculose e em sua morbidade $e$ mortalidade. 0 risco da infecção tuberculosa progredir para a doença é de aproximadamente $10 \%$ ao longo da vida do indivíduo imunocompetente. $\mathrm{Na}$ presença da coinfecção com o HIV, essa progressão é estimada em $10 \%$ ao ano. Os indivíduos com tuberculose e com infecção pelo HIV apresentam taxas de mortalidade 2,4 a 19,0 vezes mais elevadas que os sem a coinfecção. Isto se deve ao desenvolvimento da multirresistência aos tuberculostáticos, o que agrava a situação dos doentes, amplia o tempo necessário para 0 tratamento e, consequentemente, eleva os custos das ações de controle $e^{1,4}$.

Assim como outras infecções, incluindo as não oportunistas e as imunizações, a tuberculose, frequentemente promove 0 fenômeno de transativação heteróloga do HIV, levando à elevação transitória da carga viral e diminuição da contagem de linfócitos T CD4+, diminuição essa que pode também ocorrer por ação direta do $M$. tuberculosis. Estas alterações dificultam a interpretação destes parâmetros para início e/ou seleção do esquema antiretroviral. Estudos recentes sugerem que o tratamento da tuberculose em pacientes coinfectados reduz, por si só, a carga viral plasmática do HIV, mesmo nos pacientes que não estão recebendo TARV ${ }^{4,5}$.

A comorbidade TB/HIV acirra a convivência dos pacientes com as duas doenças e requer terapêutica adicional, que traz consigo novos efeitos colaterais e interações medicamentosas, demandando práticas e estratégias na assistência específicas para o acompanhamento e adesão ao tratamento. Trata-se de duas enfermidades que $a$ priori se aproximam pelo caráter de cronicidade e, portanto, da necessidade de cuidados específicos e de longo tempo ${ }^{6}$.

Assim, objetivou-se analisar, a partir da literatura, os aspectos epidemiológicos da coinfecção Tuberculose/HIV (TB/HIV) no Brasil.

\section{MÉTODOS}

Adotou-se a revisão Integrativa (RI). As seguintes fases foram percorridas para o desenvolvimento do estudo: identificação do tema e seleção da questão norteadora, amostragem ou busca na literatura, identificação dos estudos selecionados, categorização, avaliação dos estudos, interpretação dos resultados e a síntese do conhecimento evidenciado ou apresentação da revisão integrativa ${ }^{7}$.

A pergunta norteadora do processo revisional foi construída através da estratégia PICO ( $P=$ Paciente ou Problema, I=Intervenção, $\mathrm{C}=$ Comparação ou controle, $\mathrm{O}=$ Outcomes ou desfechos) e consistiu em: Qual a prevalência de coinfecção tuberculose/HIV no Brasil? ${ }^{8}$. 
Miranda LO et al

Para o levantamento bibliográfico, optou-se pela busca de artigos em periódicos nacionais e internacionais, no período de 2013 a 2017, disponíveis nas bases de dados pertencentes à Biblioteca Virtual em Saúde (BVS) - Literatura Latino-Americana e do Caribe em Ciências da Saúde (LILACS) e Medical Literature Analysis and Retrieval System on-line (MEDLINE), além do PubMed. Os descritores utilizados neste estudo foram os controlados, ou seja, aqueles estruturados e organizados para facilitar o acesso à informação: coinfecção (coinfection),

$H I V$, tuberculosis (tuberculose) e Brazil (Brasil).

Os critérios de inclusão dos estudos selecionados foram: aqueles publicados em inglês e português; primários que abordassem a temática da coinfecção tuberculose/HIV, disponíveis na íntegra de forma eletrônica e que correspondessem à faixa temporal de 2013 a 2017. Os critérios de exclusão adotados foram: impossibilidade de aquisição do artigo na íntegra e/ ou aqueles que abordassem a coinfecção TB/HIV em outros países além do Brasil, ou que não abordassem de forma conjunta a tuberculose e HIV.

Para a análise dos dados, foi utilizado 0 software IRaMuTeQ (Interface de $R$ pour lês Analyses Multidimensionnelles de Textes et de Questionnaires). Este programa informático viabiliza diferentes tipos de análise de dados textuais, desde aquelas bem simples, como a lexicografia básica (cálculo de frequência de palavras), até análises multivariadas (classificação hierárquica descendente, análises de similitude). Ele organiza a distribuição do vocabulário de forma facilmente compreensível e visualmente clara (análise de similitude e nuvem de palavras) ${ }^{9}$.

Para a continuação da realização do estudo, após os critérios de inclusão, foram selecionados 23 artigos para o processamento e análise. Para a construção do corpus, foram utilizados dados dos resultados e conclusões de cada artigo, sendo colocados em um único arquivo de texto, conforme o tutorial do IRAMUTEQ 9 .
Coinfecção Tuberculose/HIV no Brasil

Com relação à preparação do corpus, fizeram-se análises dos artigos selecionados, seguidas de correções e codificações das variáveis fixas utilizadas: Número do artigo, base de dados e ano de publicação. As análises organizadas dos artigos selecionados possibilitaram alcançar o propósito do estudo sobre coinfecções tuberculose/HIV no Brasil, sendo que os resultados foram expostos e analisados à luz do referencial teórico.

\section{RESULTADOS}

Para os 23 manuscritos, de acordo com as variáveis do estudo (número do artigo, base de dados e ano de publicação), a Classificação Hierárquica Descendente (CHD) identificou cinco classes semânticas conforme a análise dos domínios textuais e interpretação dos significados, resultando nas classes: 1 - Métodos de profilaxia para tuberculose em pacientes HIV positivos; 2 Serviços governamentais de controle à coinfecção TB/HIV; 3 - Principal fator de morte por TB/HIV; 4Coinfecção TB/HIV com maior incidência no sexo masculino; 5- Diagnóstico de tuberculose em pacientes HIV positivos. Conforme a análise lexical do material textual, a análise hierárquica descendente distribuiu as classes de acordo com o seguinte dendograma (Figura 1).

\section{Classes e suas descrições}

Classe 1. Métodos de profilaxia para tuberculose em pacientes HIV positivos

A classe 1 apresenta 38 UCEs, correspondendo a $17,76 \%$ do corpus. Os vocábulos mais frequentes e significativos destes segmentos de textos são: preventivo, isoniazida, reduzir, uso, terapia, antirretroviral, vida, qualidade $(\mathrm{p}<$ 0,0001), extraídas por ordem de significância dos artigos $23,18,8$ e 21 , conforme classificados no quadro 1. 


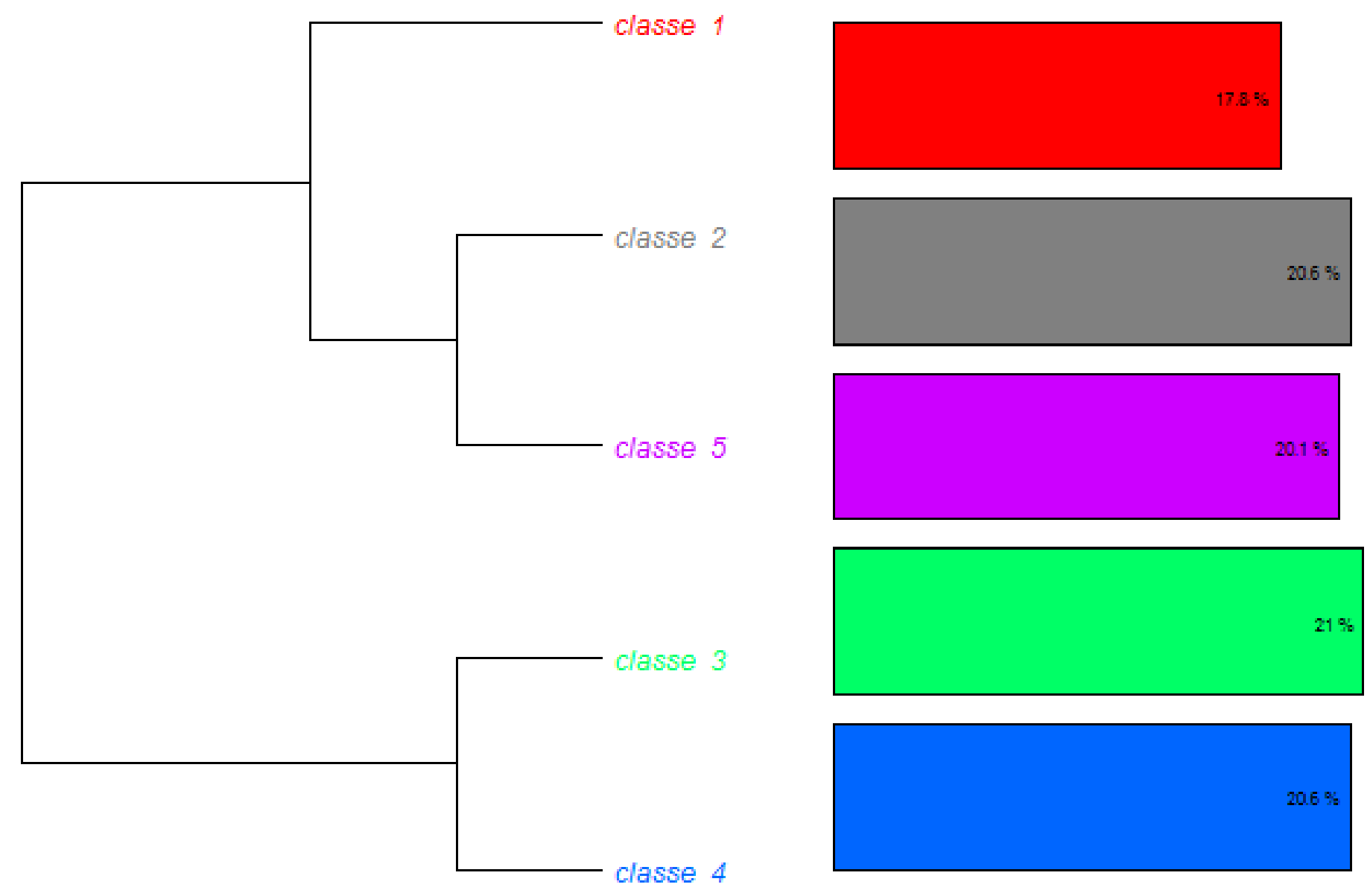

Figura 1: Classificação Hierárquica Descendente. Dendograma da coinfecção tuberculose/HIV no Brasil e aspectos epidemiológicos.

\section{Classe 2: Serviços governamentais de controle à} coinfecção TB/HIV

A classe 2 apresenta 44 UCE's de 214, correspondendo a $20,56 \%$ do corpus e está associada diretamente à classe 5 . As expressões mais frequentes destes segmentos de textos são: saúde, serviço, controle, programa, medida, governo, integração $(p<0,0001)$, extraídas predominantemente dos artigos 4, 10, 16 e 7, por ordem de significância, como segue o Quadro 1.

\section{Classe 3: Principal fator de morte por HIV/TB}

A classe 3 apresenta 45 UCE's de 214, respondendo por $21.03 \%$ do corpus e está associada diretamente à classe 4 . Os vocábulos mais frequentes e significativos destes segmentos de textos são morrer, abandono, associado, paciente, significativo $\quad(p<0,0001), \quad$ extraídas predominantemente dos artigos 17, 9, 2 e 7, por ordem de significância.
Classe 4: Coinfecção TB/HIV com maior incidência no sexo masculino

A classe 4 apresenta 44 UCEs, correspondendo a $20.56 \%$ do corpus e está associada diretamente a classe 3 . Os vocábulos mais frequentes e significativos destes segmentos de textos são: masculino, sexo, homem, médio, caso, notificar $(p<0,0001)$, extraídas predominantemente dos artigos 20, 3 e 19, por ordem de significância, de acordo com o quadro 1.

Classe 5. Diagnóstico de tuberculose em pacientes HIV positivos.

A classe 5 apresenta 43 UCEs de 214, correspondendo a $20.09 \%$ do corpus e está associada diretamente a classe 2 . Os vocábulos mais frequentes e significativos destes segmentos de textos são exame, resultado, baciloscopia, sorológico, positivo, teste, escarro $(p<0,0001)$, extraídas predominantemente dos artigos 13, 6, 11, 12 e 5, por ordem de significância, conforme o quadro 1. 
Quadro 1 - Artigos que colaboraram para a composição do corpus, conforme o IRaMuTeQ.

\begin{tabular}{|c|c|c|c|}
\hline $\begin{array}{l}\text { Número } \\
\text { do artigo }\end{array}$ & Título do artigo & Periódico & Ano \\
\hline 1 & $\begin{array}{l}\text { Factors associated with tuberculosis by HIV status in the } \\
\text { Brazilian national surveillance system: a cross sectional } \\
\text { study }\end{array}$ & $\begin{array}{l}\text { BMC Infectious } \\
\text { Diseases }\end{array}$ & 2014 \\
\hline 2 & $\begin{array}{l}\text { HIV testing, antiretroviral therapy, and treatment } \\
\text { outcomes in new cases of tuberculosis in Brazil, } 2011\end{array}$ & $\begin{array}{l}\text { Revista } \\
\text { Panamericana } \\
\text { de Salud Pública }\end{array}$ & 2016 \\
\hline 3 & $\begin{array}{c}\text { Distribuição da tuberculose em Porto } \\
\text { Alegre: análise da magnitude e coinfecção } \\
\text { tuberculose-HIV }\end{array}$ & $\begin{array}{l}\text { Rev. Esc. } \\
\text { Enferm. USP }\end{array}$ & 2014 \\
\hline 4 & $\begin{array}{c}\text { Mortality in patients with HIV-1 and tuberculosis co- } \\
\text { infection in Rio de Janeiro, Brazil - associated factors and } \\
\text { causes of death. }\end{array}$ & $\begin{array}{l}\text { BMC Infectious } \\
\text { Diseases }\end{array}$ & 2017 \\
\hline 5 & $\begin{array}{c}\text { Análise temporal dos casos notificados de } \\
\text { tuberculose e de coinfecção tuberculose-HIV } \\
\text { na população brasileira no período entre } \\
2002 \text { e } 2012\end{array}$ & J Bras Pneumol. & 2016 \\
\hline 6 & $\begin{array}{l}\text { Clinical and epidemiological characteristics associated } \\
\text { with unfavorable tuberculosis treatment outcomes in TB- } \\
\text { HIV coinfected patients in Brazil: a hierarchical } \\
\text { polytomous analysis. }\end{array}$ & Braz J Infect Dis & 2017 \\
\hline 7 & $\begin{array}{l}\text { Factors Associated with Length of Hospital Stay among } \\
\text { HIV Positive and HIV Negative Patients with Tuberculosis } \\
\text { in Brazil }\end{array}$ & PLOS ONE & 2013 \\
\hline 8 & $\begin{array}{l}\text { Quality of life among people treated for tuberculosis and } \\
\text { human immunodeficiency virus in Rio de Janeiro, Brazil }\end{array}$ & $\begin{array}{l}\text { REV Int J } \\
\text { Tuberc Lung Dis }\end{array}$ & 2013 \\
\hline 9 & $\begin{array}{l}\text { Fatores associados à coinfecção tuberculose e HIV: o que } \\
\text { apontam os dados de notificação do Estado do Amazonas, } \\
\text { Brasil, 2001-2012 }\end{array}$ & $\begin{array}{l}\text { Cad. Saúde } \\
\text { Pública }\end{array}$ & 2017 \\
\hline 10 & $\begin{array}{l}\text { Os significados da comorbidade para os pacientes vivendo } \\
\text { com TB/HIV: repercussões no tratamento }\end{array}$ & $\begin{array}{l}\text { Physis Revista } \\
\text { de Saúde } \\
\text { Coletiva }\end{array}$ & 2015 \\
\hline 11 & $\begin{array}{l}\text { Tuberculosis incidence among people living with HIV/AIDS } \\
\text { with virological failure of antiretroviral therapy in } \\
\text { Salvador, Bahia, Brazil }\end{array}$ & $\begin{array}{l}\text { The Brazilian } \\
\text { Journal of } \\
\text { Infectious } \\
\text { Diseases }\end{array}$ & 2017 \\
\hline 12 & $\begin{array}{c}\text { Tuberculose, HIV e coinfecção por TB/HIV no Sistema } \\
\text { Prisional de } \\
\text { Itirapina, São Paulo, Brasil }\end{array}$ & $\begin{array}{l}\text { Rev Inst Adolfo } \\
\text { Lutz }\end{array}$ & 2013 \\
\hline 13 & $\begin{array}{l}\text { Safety and effectiveness of HAART in tuberculosis-HIV co- } \\
\text { infected patients in Brazil }\end{array}$ & $\begin{array}{l}\text { International J } \\
\text { of Tuberculosis }\end{array}$ & 2013 \\
\hline
\end{tabular}




\begin{tabular}{|c|c|c|c|}
\hline & & $\begin{array}{l}\text { and Lung } \\
\text { Disease }\end{array}$ & \\
\hline 14 & $\begin{array}{l}\text { Three months of weekly rifapentine and isoniazid for } \\
\text { treatment of Mycobacterium tuberculosis infection in } \\
\text { HIV-coinfected persons }\end{array}$ & AIDS & 2016 \\
\hline 15 & $\begin{array}{c}\text { Infecção latente por tuberculose entre } \\
\text { pessoas com HIV/AIDS, fatores associados e } \\
\text { progressão para doença ativa em município } \\
\text { no Sul do Brasil }\end{array}$ & $\begin{array}{l}\text { Cad. Saúde } \\
\text { Pública }\end{array}$ & 2017 \\
\hline 16 & $\begin{array}{l}\text { Aspectos sociais e de saúde de portadores da coinfecção } \\
\qquad \mathrm{HIV/tuberculose}\end{array}$ & Rev Rene & 2013 \\
\hline 17 & $\begin{array}{l}\text { A spatial analysis of social and economic determinants of } \\
\text { tuberculosis in Brazil }\end{array}$ & Health \&Place & 2013 \\
\hline 18 & $\begin{array}{l}\text { Controle da Tuberculose em pessoas vivendo com } \\
\qquad \text { HIV/AIDS }\end{array}$ & $\begin{array}{l}\text { Rev. Latino-Am. } \\
\text { Enfermagem }\end{array}$ & 2016 \\
\hline 19 & $\begin{array}{l}\text { Estudo epidemiológico da coinfecção tuberculose-HIV no } \\
\text { nordeste do brasil }\end{array}$ & $\frac{\underline{\text { Revista }}}{\underline{\text { Patologia }}}$ & 2014 \\
\hline 20 & Tuberculosis in Brazil: last ten years analysis - $2001-2010$ & $\begin{array}{l}\text { The Brazilian } \\
\text { Journal of } \\
\text { infectious } \\
\text { diseases }\end{array}$ & 2013 \\
\hline 21 & $\begin{array}{c}\text { Mortality related to tuberculosis-HIV/AIDS coinfection in } \\
\text { Brazil, 2000-2011: epidemiological patterns and time } \\
\text { trends. }\end{array}$ & $\begin{array}{l}\text { Cad Saude } \\
\text { Publica }\end{array}$ & 2016 \\
\hline 22 & $\begin{array}{l}\text { Surveillance of tuberculosis coinfection among HIV } \\
\text { infected patients and their CD4+ cell count profile }\end{array}$ & $\begin{array}{l}\text { Asian Pac J Trop } \\
\text { Dis }\end{array}$ & 2015 \\
\hline 23 & $\begin{array}{l}\text { Effect of improved tuberculosis screening and isoniazid } \\
\text { preventive therapy on incidence of tuberculosis and } \\
\text { death in patients with HIV in clinics in Rio de Janeiro, } \\
\text { Brazil: a stepped wedge, cluster-randomised trial }\end{array}$ & $\begin{array}{l}\text { The Lancet } \\
\text { InfectiousDiseas } \\
\text { es }\end{array}$ & 2013 \\
\hline
\end{tabular}

Segundo a Figura 2, a nuvem de palavras gerada pelo IRaMuTeQ organiza as expressões de acordo com a sua frequência, possibilitando uma identificação de palavras-chave do estudo, conforme o corpus. As palavras de maior significância foram tuberculose, HIV, coinfecção, paciente, homem, caso e taxa. Pode-se compreender por meio dos vocábulos, que a maior prevalência de coinfecção tuberculose/HIV ocorre em pacientes do sexo masculino.
A partir do resultado produzido pelo gráfico, que gerou um leque semântico de palavras que haviam aparecido com mais frequência no corpus, as expressões mais notórias foram tuberculose, HIV, homem, paciente, coinfecção. Com essa análise complementar de similitude, entende-se que ocorrem altas taxas de tuberculose em pacientes com HIV. 


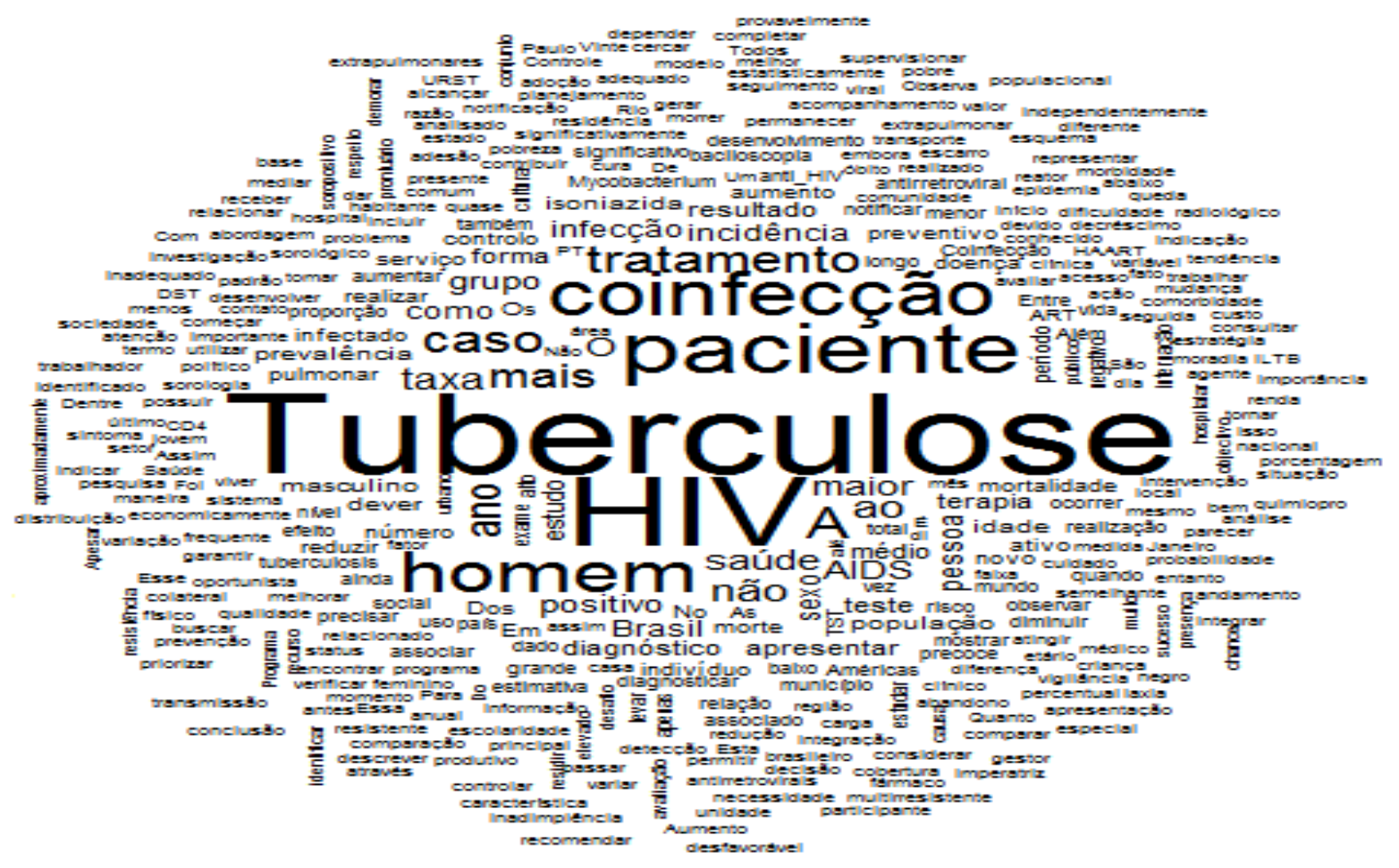

Figura 2: Nuvem de palavras. A coinfecção tuberculose/HIV no Brasil e aspectos epidemiológicos.

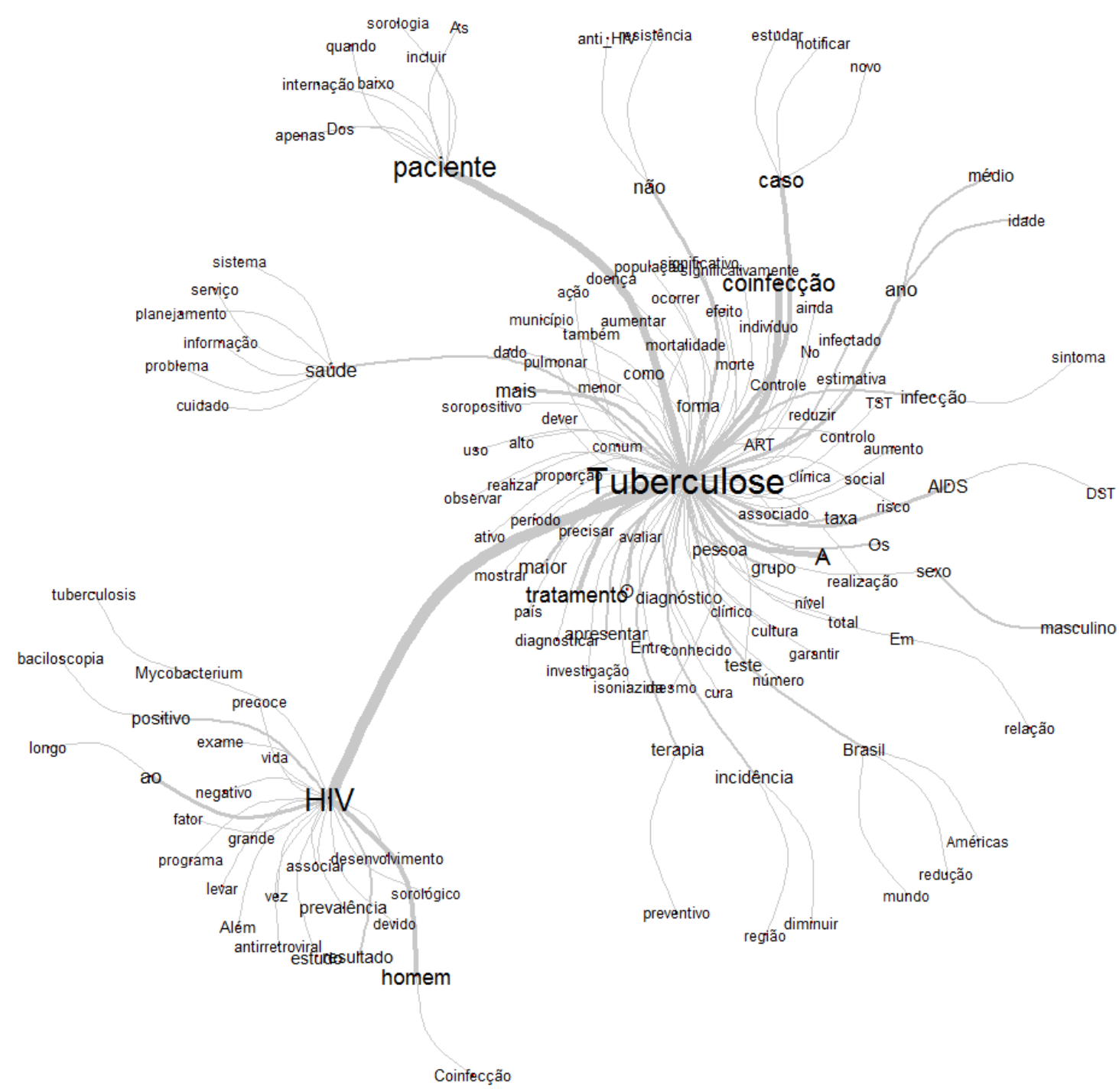

Figura 1: Análise de similitude - A coinfecção tuberculose/HIV no Brasil e aspectos epidemiológicos. 
Miranda LO et al

\section{DISCUSSÃO}

O Ministério da Saúde recomenda que todos os pacientes com tuberculose sejam submetidos à testagem sorológica para o HIV. Tal procedimento possibilita um diagnóstico precoce da infecção pelo HIV, permitindo, quando indicado, o início da terapia antirretroviral, gerando uma consequente diminuição de sua morbidade e mortalidade e constituindo-se em uma importante ferramenta para a elaboração e execução de políticas públicas $^{3}$.

A prevalência da coinfecção TB/HIV no Brasil foi associada ao sexo masculino, cor/raça negra, baixo nível de escolaridade, morar em residência urbana e idade entre 20-59 anos. O mesmo perfil epidemiológico tem sido descrito em vários estudos nas cidades brasileiras em estudos recentes ${ }^{10,11,12}$. Estas descobertas foram associadas ao estilo de vida de jovens adultos freqüentemente associados à falta de consciência de sua vulnerabilidade, expondo-os ao vírus do HIV e à tuberculose $\mathrm{e}^{13,14}$.

O perfil da coinfecção vêm mudando, apesar da grande prevalência nas regiões sul e sudeste, as taxas de incidência aumentaram nas regiões norte e nordeste, que possuem menor prevalência. $\mathrm{Na}$ Bahia, $4^{\circ}$ estado brasileiro com maior incidência de TB/HIV, pacientes portadores de HIV/AIDS com baixo nível de educação e baixa contagem células $\mathrm{CD}^{+}$estão mais propensos $\mathrm{e}$ coinfecção com a tuberculose e mortalidade precoce. No Rio de Janeiro, o mesmo resultado foi encontrado, demonstrando que a baixa contagem de células $\mathrm{CD4}^{+}$é resultado de um estado de imunodeficiência avançado com diagnóstico tardio da tuberculose. 0 acesso reduzido à informação, o conhecimento insuficiente e o acesso reduzido aos cuidados de saúde podem resultar em atraso no diagnóstico, tratamento tardio e baixa adesão ao tratamento. 0 desafio atual é integrar eficazmente os programas de controle de TB e HIV/AIDS ${ }^{15-19}$.
Coinfecção Tuberculose/HIV no Brasil

0 tratamento de pacientes coinfectados com TB-HIV é difícil. Pacientes com TB exigem tratamento de longo prazo com vários medicamentos. Para os pacientes com TB/HIV, a adesão ao regime de tratamento é difícil devido à carga extra da tomada de drogas, resultando em maior taxa de inadimplência, como mostrado em nosso estudo. Devido a esta dificuldade e à fraca adesão ao tratamento da tuberculose, devem ser planejadas novas estratégias para o monitoramento do tratamento, levando em consideração as características da coinfecção conforme recomendado pelo Plano Nacional de Controle da Tuberculose 3 ,14, 20, 21.

A taxa de cura da tuberculose na coinfecção ocorre em no máximo $50 \%$ dos casos e está relacionada com a imunodeficiência causada pelo HIV nesses pacientes, o tempo entre o diagnóstico e início do tratamento e a dificuldade de acesso aos serviços de saúde. Além disto, a taxa de abandono do tratamento chega a $13 \%$. Apesar de os tratamentos serem disponibilizados gratuitamente na rede pública, a dispensa das medicações e o seguimento do tratamento são realizados em locais distintos. Ademais, a longa duração e os efeitos colaterais do tratamento também favorecem a falta de adesão ${ }^{15,22,23}$.

O uso da TARV pode prevenir mortes entre coinfectados TB/HIV, desta forma, seu uso deve ser implementado o mais rápido possível nos indivíduos diagnosticados com HIV. No entanto, o uso isolado da terapia não garante a efetiva prevenção da coinfecção TB/HIV. Ainda assim, no Brasil, somente metade das pessoas com a coinfecção estão em uso de TARV. Santos et al. (2013) encontrou que o uso concomitante de TARV e um regime baseado em rifampicina para TB está associado a uma redução nas mortes, independentemente do regime de TARV 24, 25, 11, 26 .

O Plano Nacional de Controle da Tuberculose propõe que todos os pacientes com tuberculose ativa devem ser submetidos ao teste anti-HIV, possibilitando o início precoce da terapia 
Miranda LO et al

antirretroviral e a profilaxia das infecções oportunistas, já que a tuberculose pode acelerar o curso da doença. Apesar desta orientação, o que se observa é uma baixa frequência de solicitação e realização da sorologia, o que aumenta a incerteza sobre a real magnitude desta associação. Áreas com pior estruturação dos serviços de saúde podem subestimar os consideráveis índices de coinfecção ${ }^{4}$ 27,28 .

Ao analisarmos os óbitos por tuberculose, nota-se uma elevada taxa em coinfectados por tuberculose/HIV, podendo chegar a 20\%, sendo essa quase três vezes maior do que aquela em pacientes soronegativos. Esses dados assemelhamse aos encontrados em outros estudos e, mais uma vez, relacionam-se ao perfil imunológico de pacientes soropositivos e às taxas de abandono, maiores nessa população ${ }^{15,4}$.

0 acompanhamento epidemiológico abordando pacientes com TB internados em hospitais de referência em Manaus, foi realizado, no período de janeiro a dezembro de 2010 para detectar fatores associados ao tempo de permanência hospitalar entre HIV positivos e pacientes HIV negativos com Tuberculose no Brasil. Não houve diferenças significativas no tempo de internação em pacientes HIV positivos, todos os fatores significativamente associados à internação prolongada ocorreram neste grupo de pacientes. Este achado corrobora outros estudos que indicam a gravidade da tuberculose em pacientes com HIV, o que também pode contribuir para prolongar a sua estadia no hospital ${ }^{29}$.

Entretanto, quando observado o tratamento da TB, é possível verificar que mais de $80 \%$ dos casos adotou o regime inicial de 6 meses, usado para tratar a forma TB pulmonar. No entanto, quando observamos a distribuição de outros esquemas, é possível notar que isso ocorreu entre os pacientes HIV positivos que precisam de longos períodos de internação. É claro, o tratamento não é responsável pelo tempo de permanência hospitalar, mas podemos entender que esta
Coinfecção Tuberculose/HIV no Brasil

salienta a gravidade dos casos, especialmente aqueles entre os pacientes HIV positivos. Isso indica que os pacientes HIV positivos precisam de um esquema alternativo e talvez isso tem uma influência sobre o tempo de internação hospitalar. Ainda, existe um decréscimo sobre a qualidade de vida de pacientes vivendo com TB e HIV, mas sem diferença para pacientes com TB/HIV 29,30 .

\section{CONCLUSÃO}

Nessa revisão integrativa pode-se observar que a coinfecção HIV e tuberculose constitui um importante problema de saúde pública no Brasil. Dos artigos analisados pode-se concluir que grande número de HIV positivos desenvolve tuberculose, a coinfecção HIV/TB está associada ao sexo masculino o que os autores associam ao abandono do tratamento, não concluir o tratamento inicialmente leva ao decréscimo da qualidade de vida e posteriormente a morte e a proporção de coinfecção HIV/TB são fortemente influenciadas pelas características sócio demográficas.

Um dos maiores problemas no tratamento, tanto da HIV/AIDS, quanto da tuberculose, é a não adesão ao tratamento e/ou o abandono do mesmo, que incrementam as falhas terapêuticas e a resistência às drogas utilizadas. Muitos são os fatores que contribuem para isso, entre eles podemos citar a não aceitação da doença, o nível socioeconômico do indivíduo, a baixa escolaridade, o preconceito. É sem dúvida essencial a participação ativa do paciente no tratamento, pois o mesmo é um dos responsáveis diretos e incontestáveis da eficácia terapêutica.

\section{REFERÊNCIAS}

1. Joint United Nations Programme on HIV/AIDS (UNAIDS). GLOBAL AIDS UPDATE 2016 [Internet], 2016: 17 (Suppl. 4): 3-11. Available from:

http://pesquisa.bvsalud.org/portal/resource/pt/m $\mathrm{dl}-15080170$ 
Miranda LO et al

2. World Health Organization (WHO). Global tuberculosis report. WHO report 2016. [Internet]. Geneva: World Health Organization; 2016. [citado em 2017 set 28]. Available from:

http://www.who.int/tb/publications/global_repor t/gtbr2016_executive_summary.pdf?ua1

3. Ministério da Saúde (BR). Indicadores prioritários para o monitoramento do Plano Nacional pelo Fim da Tuberculose como Problema de Saúde Pública no Brasil. Bol Epidemiol

[Internet]. Brasília: Ministério da Saúde; 2017 [citado 2017 set 28]. Available from: http: / / portalsaude.saude.gov.br/images/pdf/2016 /marco/24/2016-009-Tuberculose-001.pdf.

4. Barbosa IR, Costa ICC. Estudo epidemiológico da Coinfecção Tuberculose-HIV no Nordeste do Brasil. Rev Patol Trop. 2014 jan-mar [cited 2017 set 02]; 43 (1): 27-38. Available from: https: //www.revistas.ufg.br/iptsp/article/view/29 $369 / 16316$

5. Novotny T, Hendrickson E, Soares ECC, Sereno AB, Kiene SM. HIV/AIDS, tuberculosis, y tabaco en Brasil: una sindemia que apela a intervenciones integ. Cad. Saúde Pública [Internet]. 2017 [cited 2017 Oct 07]; 33(Suppl 3): e00124215. Available from:

http: / / www.scielosp.org/scielo.php?script=sci_artt ext\&pid=S0102-311X2017001100301\&lng=en

6. Silva JB, Cardoso GCP, Netto AR, Kritski AL. Os significados da comorbidade para os pacientes vivendo com TB/HIV: repercussões no tratamento. Physis Revista de Saúde Coletiva. 2015 [cited 2017 set 14]; 25(1): 209-229. Available from: http: / / www.scielo.br/pdf/physis/v25n1/01037331-physis-25-01-00209.pdf

7. Mendes KDS, Silveira RCCP, Galvão CM. Revisão integrativa: método de pesquisa para a incorporação de evidências na saúde e na enfermagem. Texto contexto - enferm. [Internet]. 2008 Dec [cited 2017 Oct 08]; 17(4):758-764. Available from: http: / / www.scielo.br/scielo.php?script=sci_arttext \&pid=S0104-07072008000400018\&lng=en
Coinfecção Tuberculose/HIV no Brasil

8. Santos CMC, Pimenta CAM, Nobre MRC. The PICO strategy for the research question construction and evidence search. Rev. Latino-Am. Enfermagem [Internet]. 2007 June [cited 2017 Oct 08]; 15(3):508-511. Available from:

http: / / www.scielo.br/scielo.php?script=sci_arttext \&pid=S0104-11692007000300023\&lng=en

9. Camargo BV, Justo AM. IRAMUTEQ: Um software gratuito para análise de dados textuais. Temas em Psicologia [Internet]. $2013 \mathrm{dec}$ [cited 2017 oct 02]; 21(2), 513-518. Available from: http://pepsic.bvsalud.org/scielo.php?script=sci_art text\&pid=S1413-389X2013000200016

10. Lemos LA, Feijão AR, Galvão MTG. Social and health aspects of patients co-infected with HIV/tuberculosis. Rev Rene [Internet]. 2013 [cited 2017 sep 14]; 14(2):364-71. Available from: http://www.revistarene.ufc.br/revista/index.php/ revista/article/view/805

11. Torrens A, Bartholomay P, Silva S, Khogali M, Verdonck K, Bissell K. HIV testing, antiretroviral therapy, and treatment outcomes in new cases of tuberculosis in Brazil, 2011. Rev Panam Salud Publica [Internet]. 2016 Jan [cited 2017 Oct 07]; 39(1):26-31. Available from:

http: / / www.scielosp.org/scielo.php?script=sci_artt ext\&pid=S1020-49892016000100026\&lng=en.

12. Magno ES, Saraceni V, Souza AB, Magno RS, Saraiva MGG, Bührer-Sékula S. Fatores associados à coinfecção tuberculose e HIV: o que apontam os dados de notificação do Estado do Amazonas, Brasil, 2001-2012. Cad. Saúde Pública [Internet]. 2017 [cited 2017 Oct 07]; 33(5):e00019315.

Available from:

http: / / www.scielo.br/scielo.php?script=sci_arttext \&pid=S0102-311X2017000505006\&lng=en

13. Prado D, Miranda A, Souza F, Dias E, Sousa L, Arakaki-Sanchez D, Sanchez M, Golu J, Maciel J. Factors associated with tuberculosis by HIV status in the Brazilian national surveillance system: a cross sectional study. BMC Infect Dis [Internet]. 2014 [cited 2017 Oct 07]; 14:415. 
Miranda LO et al

https://www.ncbi.nlm.nih.gov/pmc/articles/PMC4 $122782 /$

14. Oliveira GP, Torrens AW, Bartholomay P, Barreira D. Tuberculosis in Brazil: last ten years analysis - 2001-2010. Braz J Infect Dis [Internet]. 2013 Apr [cited 2017 Oct 07]; 17(2): 218-233. Available from:

http: / / www.scielo.br/scielo.php?script=sci_arttext \&pid=S1413-86702013000200016\&lng=en

15. Gaspar RS, Nunes N, Nunes M, Rodrigues VP. Análise temporal dos casos notificados de tuberculose e de coinfecção tuberculose--HIV na população brasileira no período entre 2002 e 2012. J. bras. pneumol. [Internet]. 2016 Dec [cited 2017 Oct 07]; 42(6): 416-422. Available from:

http: / / www.scielo.br/scielo.php?script=sci_arttext \&pid=S1806-37132016000600416\&lng=en

16. Rebouças MC, Silva MO, Haguihara T, Brites C, Netto EM. Tuberculosis incidence among people living with HIV/AIDS with virological failure of antiretroviral therapy in Salvador, Bahia, Brazil. Braz J Infect Diseases [Internet]. 2017 [cited 2017 Oct 07]; 21(5): 562-566. Available from:

http://www.sciencedirect.com/science/article/pii /S1413867017301526?via\%3Dihub

17. Escada RO, Velasque L, Ribeiro SR, Cardoso SW, Marins LMS, Grinsztejn E, et al. Mortality in patients with HIV-1 and tuberculosis co-infection in Rio de Janeiro, Brazil - associated factors and causes of death. BMC Infect Dis [Internet]. 2017 May [cited 2017 Oct 07]; 17(1):373. Available from: https://www.ncbi.nlm.nih.gov/pmc/articles/PMC5 450415/

18. Lima MS, Martins-Melo FR, Heukelbach J, Alencar CH, Boigny RN, Ramos JAN. Mortality related to tuberculosis-HIV/AIDS co-infection in Brazil, 2000-2011: epidemiological patterns and time trends. Cad. Saúde Pública [Internet]. 2016 Oct [cited 2017 Oct 07]; 32(10): e00026715. Available from: http: / / www.scielo.br/scielo.php?script=sci_arttext \&pid=S0102-311X2016001005008\&lng=en
Coinfecção Tuberculose/HIV no Brasil

19. Jain S, Singh AK, Singh RP, Bajaj J, Damlec

AS. Surveillance of tuberculosis co-infection among HIV infected patients and their CD4+ cell count profile. Asian Pacific Journal of Tropical Diseases. 2015 March [cited 2017 Oc 01]; 5(3):234-238.

Available from:

http://www.sciencedirect.com/science/article/pii /S2222180814606605

20. Prado TN, Rajan JV, Miranda AE, Dias ES, Cosme LB, Possuelo LG, et al. Clinical and epidemiological characteristics associated with unfavorable tuberculosis treatment outcomes in TB-HIV co-infected patients in Brazil: a hierarchical polytomous analysis. Braz J Infect Dis [Internet]. 2017 Mar [cited 2017 Oct 08]; 21(2): 162-170. Available from:

http: / /www.scielo.br/scielo.php?script=sci_arttext \&pid=S1413-86702017000200162\&lng=en

21. Durovni B, Saraceni V, Moulton LH, Pacheco AG, Cavalcante SC, King BS, et al. Effect of improved tuberculosis screening and isoniazid preventive therapy on incidence of tuberculosis and death in patients with HIV in clinics in Rio de Janeiro, Brazil: a stepped wedge, clusterrandomised trial. Lancet Infect Dis [Internet]. 2013 oct [cited 2017 Oct 01];13(10): 852-858. Available from:

https://www.ncbi.nlm.nih.gov/pubmed/23954450

22. Santos DT, Garcia MC, Costa AANF, Pieri FM, Meier DAP, Albanese SPR, et al. Infecção latente por tuberculose entre pessoas com HIV/AIDS, fatores associados e progressão para doença ativa em município no Sul do Brasil. Cad. Saúde Pública [Internet]. 2017 [cited 2017 Oct 08]; 33(8): e00050916. Available from: http: / /www.scielo.br/scielo.php?script=sci_arttext \&pid=S0102-311X2017000805008\&lng=en

23. Harling G, Castro MC. A spatial analysis of social and economic determinants of tuberculosis in Brazil. Health Place [Internet]. 2013 [cited 2017 Oct 08]; 25: 56-67. Available from:

http://www.sciencedirect.com/science/article/pii /S135382921300141X?via\%3Dihub 
Miranda LO et al

24. Santos APG, Pacheco AG, Staviack A, Golub JE, Chaisson RE, et al. Safety and effectiveness of HAART in tuberculosis-HIV co-infected patients in Brazil. International Journal of Tuberculosis and Lung Disease [Internet]. 2013 [cited 2017 Oct 08]; 17(2): 192-197. Available from:

https://www.ncbi.nlm.nih.gov/pmc/articles/PMC3 713776/

25. Magnabosco GT, Lopes LM, Andrade RLP, Brunello MEF, Monroe AA, Villa TCS. Controle da Tuberculose em pessoas vivendo com HIV/aids. Rev. Latino-Am. Enfermagem [Internet]. 2016 [cited 2017 Oct 08]; 24: e2798. Available from: http: / / www.scielo.br/scielo.php?script=sci_arttext \&pid=S0104-11692016000100410\&lng=en

26. Peruhype RC, Acosta LMW, Ruffino NA, Oliveira MMC, Palha PF. Distribuição da tuberculose em Porto Alegre: análise da magnitude e coinfecção tuberculose-HIV. Rev. esc. enferm. USP [Internet]. 2014 Dec [cited 2017 Oct 08]; 48(6): 1035-1043. Available from:

http: / / www.scielo.br/scielo.php?script=sci_arttext \&pid=S0080-62342014000601035\&lng=en

27. Sterling TR, Scott NA, Miro JM, Calvet G, LaRosa A, Infante R, et al; Tuberculosis Trials Consortium, the AIDS Clinical Trials Group for the PREVENT TB Trial (TBTC Study 26ACTG 5259).

Three months of weekly rifapentine and isoniazid

\section{COLABORAÇÕES}

Miranda LO, Araujo GBF, Carvalho ML, Silva SMF, Andrade DFR, Moreira AM, Freitas DRJ contribuíram na coleta e interpretação dos resultados obtidos e ainda, na elaboração e organização das ideias e nas revisões sucessivas até a aprovação final.

\section{CONFLITOS DE INTERESSE}

Não há conflitos de interesse a declarar

\section{CORRESPONDENCIA}

Daniela Reis Joaquim de Freitas

Universidade Federal do Piauí

Campus Universitário Ministro Petrônio Portella, s/n - Ininga, Teresina - PI, 64049-550

E-mail: dr danielarjfreitas@ufpi.edu.br for treatment of Mycobacterium tuberculosis infection in HIV-coinfected persons. AIDS [Internet]. 2016 jun [cited 2017 Oct 01]; 30(10):1607-1615. Available from:

28. Aily DCG, Berra JAP, Brandão AP, Chimara

E. Tuberculose, HIV e coinfecção por TB/HIV no Sistema Prisional de Itirapina, São Paulo, Brasil. Rev inst adolfo lutz [Internet]. 2013 [cited 2017 Sept 23]; 72(4): 288-94. Available from: http://revistas.bvsvet.org.br/rialutz/article/view/ 22894

29. Goncalves MJ, Ferreira AA. Factors associated with length of hospital stay among HIV positive and HIV negative patients with tuberculosis in Brazil. PLoS One [Internet]. 2013 [cited 2017 Oct 03]; 8(4): e60487. Available from: https://www.ncbi.nlm.nih.gov/pubmed/23593227 30. Dowdy DW, Israel G, Vellozo V, Saraceni V, Cohn S, Cavalcante S, et al. Quality of life among people treated for tuberculosis and human immunodeficiency virus in Rio de Janeiro, Brazil. Int J Tuberc Lung Dis [Internet]. 2013 [cited 2017 Oct 03]; 17(3): 345-7. Available from: https://www.ncbi.nlm.nih.gov/pmc/articles/PMC3 $718256 /$ https://www.ncbi.nlm.nih.gov/pubmed/27243774 